\title{
PHOTOELECTRIC SPECTROPHOTOMETRY BY THE NULL METHOD ${ }^{1}$
}

\author{
By K. S. Gibson
}

CONTENTS

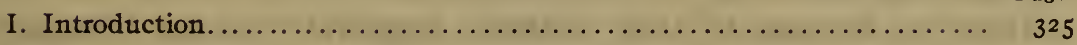

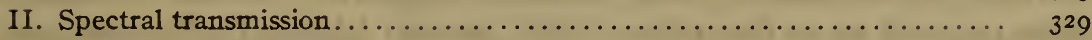

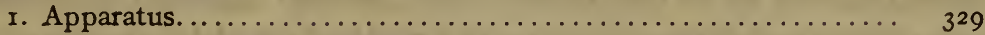

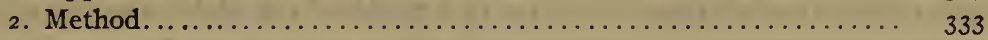

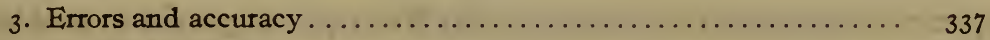

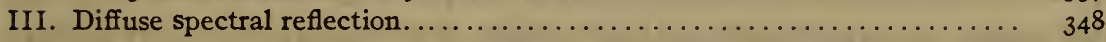

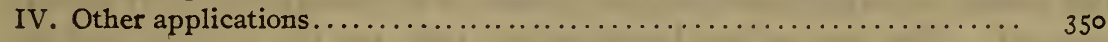

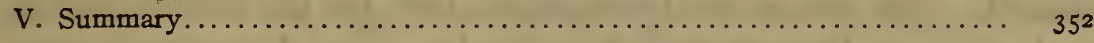

\section{INTRODUCTION}

Anyone who has had experience in trying to make spectrophotometric measurements of transmission or reflection in the blue and violet parts of the spectrum is well aware of the difficulty of obtaining reliable determinations in this region. Nearly all methods have relatively low sensitivity, or else are inaccurate for other reasons, from wave lengths 400 to 500 millimicrons $(\mathrm{m} \mu)$. Radiometric methods, which are so suitable for infra-red work and which have been used also in the visible and even in the ultraviolet, are, nevertheless, not of the highest accuracy in these latter regions because of the relatively low radiant power of all sources used for this kind of work, the radiant power decreasing continuously with the wave length. It is very difficult to obtain accurate determinations below $500 \mathrm{~m} \mu$ by the usual radiometric methods. Any visual method is limited in the blue and violet because of the combined low visibility of the human eye and the low radiant power of the sources used, except at the few wave lengths where monochromatic light of great intensity can be obtained, as from the mercury arc. The Hilger sector photometer method, which is the only photographic method of speed and reliability, also has its limitations for this region. Being such a

${ }^{1}$ An abst-act of this paper was presented to the Optical Society of America at the Baltimore meeting, Dec., 27, 1918. 
practicable method for the ultra-violet, it is always used in connection with a quartz spectrograph, and the low dispersion thus obtained in the violet and blue makes it difficult to obtain reproducible values at these wave lengths. Moreover, it is to a certain extent unreliable at high transmissions. In a recent paper ${ }^{2}$ some examples are given of the kind of agreements and disagreements obtained from 400 to $500 \mathrm{~m} \mu$ when the transmissions of good specimens were measured by visual (König-Martens and LummerBrodhun spectrophotometers and Martens photometer with monochromatic light) and photographic (Hilger sector photometer) methods.

However, because of their extreme sensitivity to the blue and violet, as well as to the ultra-violet, certain kinds of photoelectric cells offer possibilities of devising a method for obtaining as accurate spectrophotometric measurements from 400 to $500 \mathrm{~m} \mu$, and beyond this range, as are obtained at any other wave lengths by any other method. The potassium-hydride cell now on the market, when used with an incandescent lamp and a glass dispersing prism, gives a maximum response usually near $460 \mathrm{~m} \mu$; and the photoelectric method, under these conditions, admirably supplements the visual and photographic methods, being best where they become poor and becoming poor only after they have become reliable.

In connection with the color-standardization work of the National Bureau of Standards, ${ }^{3}$ it is desired to have available a number of independent methods of making spectrophotometric determinations, especially in the visible part of the spectrum; for it is generally admitted that the fundamental basis of color specification is spectrophotometry. To supplement the other methods at present in use at the Bureau, and especially to overcome the previously mentioned uncertainty of measurements made in the blue and violet, the writer in 1917 was given the problem of developing a method for accurate and convenient photoelectric spectrophotometry suitable for routine determinations. A null method was decided upon for reasons which will be explained later, an electrometer serving merely as an indicator of equality between two photoelectric currents. The making and assembling of apparatus was completed in April, 1918; and since that time it has been in continual use, being very satisfactory as

2 Gibson and McNicholas, The Ultra-Violet and Visible Transmission of Eye-Protective Glasses, Bureau of Standards Tech. Paper, No. IIg, June, xgrg.

${ }^{8}$ I. G. Priest, The Work of the National Bureau of Standards on the Establishment of Color Standards and Methods of Color Specifications, Trans. I. E. S., XIII, p. 38; 1918. 
to speed of operation, ease of keeping in working condition, and reliability of measurement.

In a recent paper by Coblentz ${ }^{4}$ is given a brief summary of some of the characteristics and methods of use of the photoelectric cell, together with references to most of the papers on the subject, and no general discussion will be given herein except as serves the needs of this paper Hitherto, whenever the photoelectric cell has been used in measuring spectral transmission or reflection, and, in fact, in practically all kinds of work, some form of deflection method has been used. Any deflection method, except a method of equal deflections, is almost valueless for accurate work unless the relation between the photoelectric current and the exciting radiant power is accurately known for all values of wave length and radiant power to be used. It has been a much-discussed question whether or not this relation takes the form of a straight-line curve for any or all photoelectric cells. Suffice it here to say that it has been concluded by the majority of investigators in this field that it is not safe to assume for any photoelectric cell a direct proportionality between photoelectric current and exciting radiant power. Any cell which is to be used in a deflection method (with the exception just noted) must first be carefully tested (and calibrated if any deviation from the straight-line relationship is detected), and should be repeatedly retested to make sure that conditions have not changed and that things are always in proper working order.

Another source of trouble in most deflection methods has been the difficulty of eliminating the so-called dark current; i. e., the current through the cell when not irradiated. ${ }^{5}$ When this has not been completely eliminated or balanced, its magnitude has usually been measured and corrections made in the computations. Gradually, however, the technique of making photoelectric cells has been improved, until now most of the cells made on the "black-body" principle are found to obey very nearly the straight-line relationship, and the dark current can be much more easily suppressed.

Nevertheless, a method which eliminates any possible errors and the necessity of any tests, calibrations, or corrections due to these two factors is of much greater value and reliability than is any method into which these factors may enter.

\footnotetext{
- Instruments and Methods Used in Radiometry, III, Bureau of Standards Scientific Paper, No. 319. xgrs.

The terms irradiate and irradiation, as emphasized by H. E. Ives (Astro. Journ., XLV, p. 39; 1917). should be used analogously to the terms illuminate and illumination when radiant energy. rather than light is discussed.
} 
Either the galvanometer or the electrometer may be used with the photoelectric cell in spectrophotometry. If the radiant powers used are sufficiently great, then, of course, the galvanometer is quicker and more convenient than the electrometer. The advantage of the electrometer over the galvanometer, however, is the greater sensitivity that can be attained, which enables measurements to be made over a greater range of wave lengths and, at least for low values of transmission, with much greater accuracy. The use of the electrometer in any deflection method, however, requires considerable time and skill if accurate results are to be obtained. A strict proportionality between deflections of the disk, as read by means of the mirror and scale, and the potential acquired by the quadrants must be proved by test or a calibration made. The use of the rate-of-drift method or the total-deflection or ballistic-throw (in a given interval of time) method necessitates the use of timing devices, with their inconvenience and possible introduction of further errors. If the electrometer is shunted around a high resistance and the steady deflections read, the sensitivity is lowered.

The only means by which the questions of the current-irradiation relationship and the dark current may be avoided and their possible errors eliminated, while retaining the extreme sensitivity of the unshunted electrometer, is by the proper use of a null method, where the electrometer serves simply as an indicator of certain desired conditions.

The photoelectric null method has not heretofore been used in the measurement of spectral transmission or reflection, indeed in scarcely any kind of quantitative work. Its possibilities were first brought out by Richtmyer, ${ }^{6}$ who used two photoelectric cells and batteries in a sort of Wheatstone bridge arrangement, the electrometer serving merely as an indicator of equality between the currents through the two photoelectric cells. These currents were excited by the undispersed radiant energy from two incandescent lamps. By varying the distances of the lamps from the cells, data were obtained which showed that the cells obeyed exactly the same current-irradiation law. He pointed out that, besides eliminating all errors due to dark currents, this method makes possible, for certain kinds of work, a precision of measurement not otherwise available.

The arrangement of apparatus for the null method herein described is essentially the same as that used by Richtmyer, but 
adapted to the needs of spectrophotometry. It has so far been used for two purposes, the measurement of spectral transmission and the measurement of diffuse spectral reflection relative to that of a second substance, such as magnesium carbonate, as a standard. Other applications will be pointed out later. Inasmuch as by far the greater part of the work for which the apparatus was needed was the measurement of spectral transmission, the method was designed and the apparatus installed with this primarily in mind.

\section{SPECTRAL TRANSIMISSION}

\section{APPARATUS}

The essential parts of the apparatus ${ }^{7}$ are outlined in Fig. I, which, in connection with the key on the page opposite the figure, is practically self-explanatory. In addition the following points might be noted:

In order to eliminate so far as possible all vibrations which might affect the sensitive electrometer disk, the apparatus was set up in the basement on the ground floor, an absolutely rigid support being furnished by the concrete piers, slate slab, and cast-iron base. This arrangement has been entirely satisfactory, the electrometer being unaffected by vibrations of any kind.

The aluminum box (aluminum being used because of its light weight) with the iron base serves three purposes:

I. Being grounded, it gives complete protection to the electrometer, the photoelectric cells, and the connecting wires from all extraneous electrical disturbances.

2. It prevents any radiant energy to which the photoelectric cells are sensitive from reaching them, except what comes through the windows $W_{1}$ or $W_{2}$. The inside walls, including the partitions $N$, are painted a dull black.

3. It makes it possible to dry out thoroughly the air around the electrometer, the photoelectric cells, and the insulating materials-an absolute necessity for success. Concentrated sulphuric acid in open dishes in each one of the three compartments has been very efficient as a drying agent. Melted paraffin was poured into the lower brass channels to make them air-tight, but it has not been necessary to seal in the cover

The electrometer is adjusted so that the reading of the spot of light is about the same when the disk is charged to +150 volts as

\footnotetext{
7 The aluminum inclosure, the electrometer, the bilateral slits, the carrier arrangement, the lamp inclosures, and many other minor pieces of the apparatus were constructed in the Bureau instrument shop by J. A. Johannessen.
} 
KEX TO FIG. 1

[Apparatus drawn to scale shown in diagram except for batteries and a few other minor details.]

$F F F=$ Slate slab supported by two concrete pillars resting on basement floor.

$I I I I=$ Cast-iron base $3.5 \mathrm{~cm}$ thick resting upon slate slab.

$J J J J=$ Aluminum box $50 \mathrm{~cm}$ high, divided into three compartments by partitions $N$ and fitting into brass channels on iron base. Has aluminum cover fitting into brass channels at top of box.

$E=$ Dolezalek quadrant electrometer, resting on iron base, with aluminum disk $E^{\prime}$ suspended by platinized quartz fiber.

$P_{1} P_{2}=$ Kunz potassium-hydride photoelectric cells supported by tripods resting on iron base.

$A A A=$ Pieces of polished amber with brass rods through centers.

$G G^{\prime}=$ Ground-wire connections to water pipe. Cast-iron base, aluminum box, electrometer case, and one pair of quadrants permanently grounded.

$U=U$ tube filled with mercury into which dips a pointed amalgamated copper wire. Used for makeand-break ground connection for second pair of electrometer quadrants, controlled from $X, X^{\prime}$, or $X^{\prime \prime}$

$W W_{1} W_{2}=$ Windows.

$H=$ Hilger constant-deviation spectrometer, resting on slate slab, with glass prism and accurately adjustable bilateral slits $S_{2}$ and $S_{3}$.

$T=$ Track of iron Scimatco optical bench resting on ground floor.

$L_{2}=$ Mazda $C$ moving-picture lamp rated at 600 watts ( 30 volts, 20 amperes), filament in one plane about $\mathrm{I} \mathrm{cm}$ square, completely inclosed except for concealed ventilating openings and openings $3 \mathrm{~cm}$ in diameter in front and back of filament. $L_{2}$ is carried by a three-wheeled car moving along photometer track and carrying a marker directly beneath filament by which its distance from slit $S_{2}$ may be read on scale shown in diagram.

$Z=$ Black felt screens, supported on $T$, enabling measurements to be made with other lights in the room.

$Y=$ Leeds and Northrup rotating sector and motor $Y^{\prime}$, supported on $T$, used to test inverse-square law for $L_{2}$ and to increase range of measurements.

$C=$ Sliding carrier, supported on $T$, containing specimen $B$, whose transmission is to be measured. Carrier slides back and forth from $C$ to $C^{\prime}$.

$Q=$ Container for selective ray filters.

$L_{1}=$ Mazda $C$ automobile headlight lamp rated at ${ }_{4} 4$ watts ( 7 volts, 2 amperes), inclosure similar to that for $L_{2}$, supported by iron base. $L_{1}$ and $L_{2}$ are run in parallel with suitable adjustable resistances on a 6o-volt storage battery.

$S_{1}=$ Accurately adjustable bilateral slit, supported on iron base, identical with $S_{2}$ and $S_{2}$.

$Q^{\prime}=$ White opal glass screen.

$V=$ Battery of Eveready cells, usually about $+{ }_{50}$ volts, by which electrometer disk may be charged through conducting suspension.

$V_{1} V_{2}=$ Battery of Eveready cells, usually about 40 and 80 volts, respectively, of such value that the dark currents through $P_{1}$ and $P_{2}$ are approximately equal.

$R=$ Adjustable high resistance.

$K K_{1} K_{2}=$ Knife switches.

$L=25$-watt vacuum tungsten lamp. Light from filament is reflected and focused by small mirror on electrometer disk to give image on $50 \mathrm{~cm}$ ground-glass scale at $D$.

$X=$ Approximate position of observer when scale is at $D$.

$X^{\prime} D^{\prime}, X^{\prime \prime} D^{\prime \prime}=$ Other positions of observer and ground-glass scale under conditions explained in paper. In these cases light from $L$ is reflected to $D^{\prime}$ or $D^{\prime \prime}$ by a mirror $M^{\prime}$ or $M^{\prime \prime}$. 


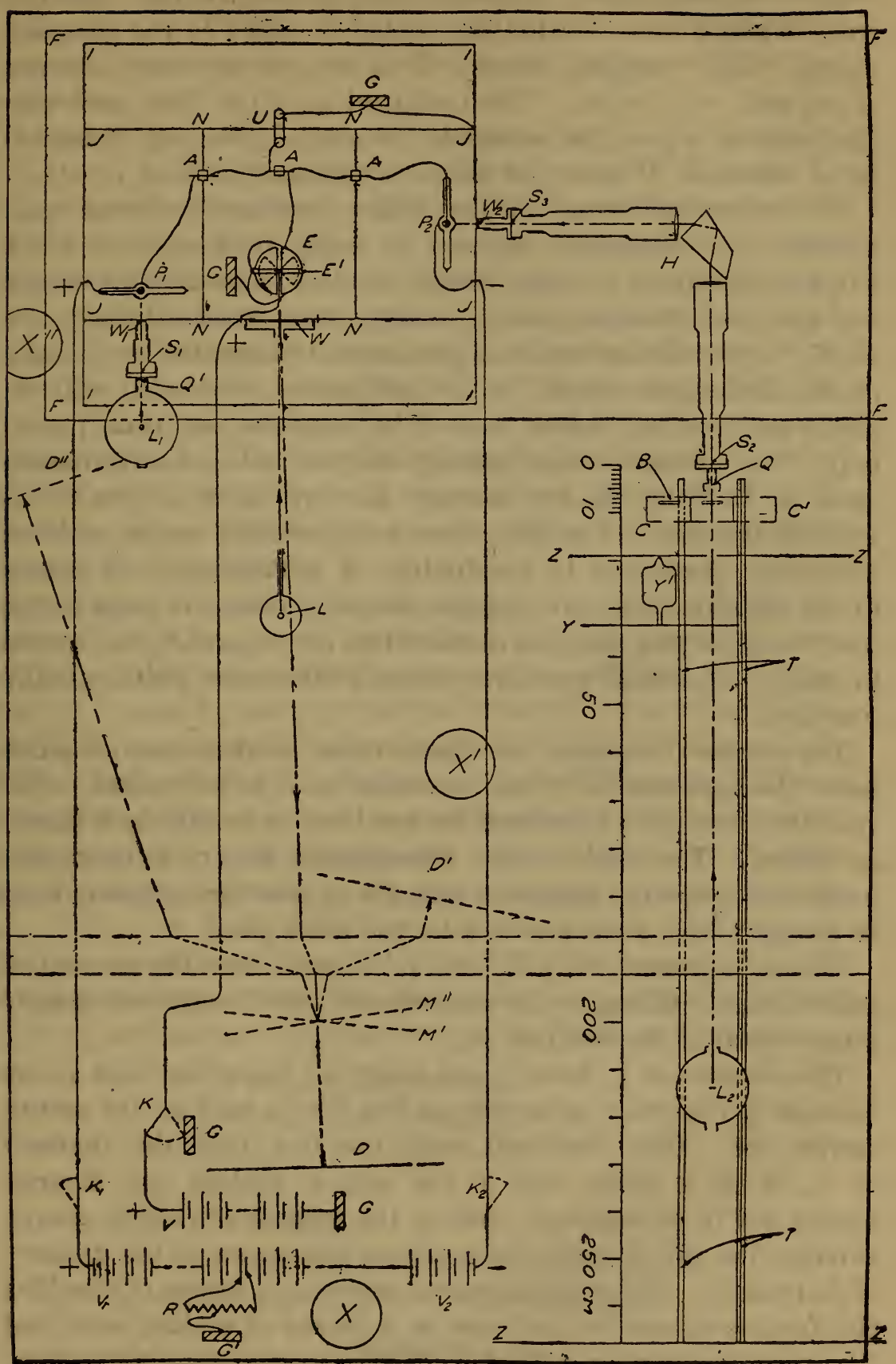

FIG. I.-Diagram of apparatus. For explanation, see opposite page $117366^{\circ}-19-2$ 
when it is uncharged. However, the position of the "charged zero," which varies with the charge, has no effect on the measurements. The charging battery $V$ is kept somewhere between $+\mathrm{I} 25$ and $+\mathrm{I} 75$ volts. When charged to $+I_{50}$ volts and with the scale at $2.5 \mathrm{~m}$, the sensitivity is about $3000 \mathrm{~mm}$ deflection for a difference of potential between the quadrants of I volt.

The prism and lenses of the Hilger constant-deviation spectrometer are completely inclosed by many thicknesses of black felt, and absolutely no stray visible or ultra-violet radiant energy can get into the spectrometer except what comes through the slit $S_{2}$. Even this has been to a great extent eliminated by specially made diaphragms placed in the collimators, and what still remains can be easily taken care of by selective ray filters placed in $Q$. The calibration was carefully checked and such adjustments made as to bring the 436 mercury line into focus at the slit $S_{3}$ without parallax. The slits were very carefully made and are accurately graduated to hundredths of millimeters. As shown in the diagram, they are stopped down by means of caps fitting over them, so that slit $S_{2}$ is in effect but $1.0 \mathrm{~cm}$ and $S_{3}$ but $2.0 \mathrm{~cm}$ in height. $S_{2}$ and $S_{3}$ are always kept at the same width, usually $0.20 \mathrm{~mm}$.

The carrier $C$ contains two hard-rubber holders, one of which holds the specimen $B$, whose transmission is to be studied, while the other may hold a comparison specimen or be simply a blank, as desired. The whole carrier arrangement is very substantially made and keeps the specimen securely in position, enabling it to be brought back always to exactly the same place.

The arrangement of $L_{1}, S_{1}$, and $Q^{\prime}$ is such that the amount of radiant energy falling on the photoelectric cell $P_{1}$ will be accurately proportional to the width of $S_{1}$.

The distance of $L_{2}$ from $S_{2}$ can never be made less than $46 \mathrm{~cm}$ because of the other apparatus on the bench, such as the sector, carrier, etc. This, combined with the fact that the filament of $L_{2}$ is in a plane only I cm square, enables the inversesquare law to be assumed; that is, the amount of radiant energy entering the slit $S_{2}$ varies inversely as the square of the distance of $L_{2}$ from $S_{2}$. The rotating sector serves as a means of checking this inverse-square law and also as a means of making sure that the apparatus keeps in perfect working condition from day to day. The length of the photometer bench enables $L_{2}$ to be moved back to $255 \mathrm{~cm}$ from the slit. Therefore, the range of transmission possible with the ro per cent sector is from r.oo to approximately 
0.004. The distance of $L_{2}$ from the slit $S_{2}$ is varied by means of a cord fastened to the ends of the car carrying $L_{2}$ and passing over pulleys at the ends of the photometer bench, and is controlled by the observer at $X$ or $X^{\prime}$.

By operating $L_{1}$ and $L_{2}$ in parallel, errors due to any fluctuating voltage are partially eliminated. These currents through $L_{1}$ and $L_{2}$ are read by ammeters and controlled by rheostats operated from the position $X^{\prime}$. When an observer works alone, he takes the position $X^{\prime}$ (the scale being at $D^{\prime}$ and the mirror at $M^{\prime}$ ), where he can watch the currents through $L_{1}$ and $L_{2}$, observe the spot of light on $D^{\prime}$, and adjust the distance of $L_{2}$, and where it is convenient for him to change the wave-length scale of $H$ and to shift the carrier $C$ back and forth. If he has an assistant, the observer takes the position $X$, where he can adjust the distance of $L_{2}$ and observe the spot of light on $D$. In this case, the assistant is at $X^{\prime}$, where he can watch the currents, adjust the wave length, and operate the carrier.

The switches $K_{1}$ and $K_{2}$ are usually kept closed even when the apparatus is not in use; and inasmuch as the dark currents do not need to be exactly balanced for transmission measurements, the values of $V_{1}$ and $V_{2}$ are very seldom changed. The value of the ratio $V_{2} / V_{1}$ necessary to balance the dark currents through $P_{1}$ and $P_{2}$ is approximately two, though this varies somewhat from time to time. The switch $K$ is used to charge the electrometer disk or to ground it when not in use. Every few weeks the batteries are tested, and those found depreciating are replaced by new ones. Otherwise $V, V_{1}$, and $V_{2}$ need no attention. Under actual operating conditions the various quantities have so far usually had the following values, these being such as to give the desired sensitivity and balance with the lamp $L_{2}$ well back on the track:

$$
\begin{aligned}
& V=+\mathrm{I} 50 \text { volts, approximately. } \\
& V_{1}=+40 \text { volts, approximately. } \\
& V_{2}=-80 \text { volts, approximately. } \\
& S_{1}=1.00 \mathrm{~mm} . \\
& S_{2}=S_{3}=0.20 \mathrm{~mm} . \\
& i_{1}=1.25-1.85 \text { amperes = current through } L_{1} . \\
& i_{2}=17.0-19.5 \text { amperes }=\text { current through } L_{2} .
\end{aligned}
$$

\section{METHOD}

The theory upon which the whole method is based is very simple, indeed. When the ground connection is made at $U$, both pairs of quadrants are at zero potential. Under this condition the 
charged electrometer disk will be at rest. Let the voltage applied to $P_{1}$ be $V_{1}$ and that applied to $P_{2}$ be $V_{2}$, and let the total resistances (mainly that of the photoelectric cells) between $U$ and $G^{\prime}$ be $R_{1}$ by way of $P_{1}$ and $R_{2}$ by way of $P_{2}$. Then through $P_{1}$ a current will flow of magnitude $I_{1}=V_{1}^{\prime} / R_{1}$ and through $P_{2}$ of magnitude $I_{2}=V_{2} / R_{2}$. No radiant energy to which the photoelectric cells are sensitive is considered as falling on them, $I_{1}$ and $I_{2}$ being what are ordinarily called the dark currents.

If the ground connection at $U$ is broken, the electrometer disk will be deflected (shown by the drift of the spot of light) unless $I_{1}$ is exactly equal to $I_{2}$. If they are not equal, they may be made so by changing the relative values of $V_{1}$ and $V_{2}$. This is done by varying the point of ground connection at $G^{\prime}$.

Now let $P_{1}$ and $P_{2}$ be irradiated by $L_{1}$ and $L_{2}$. This will bring about a great increase in the photoelectric currents $I_{1}$ and $I_{2}$, and the irradiations may be so adjusted (by varying the currents $i_{1}$ or $i_{2}$, the width of $S_{1}$, or the distance of $L_{2}$ ) as to make $I_{1}$ again exactly equal to $I_{2}$, as shown by the zero motion of the electrometer disk when the ground connection at $U$ is broken. Now let the irradiation of $P_{2}$ from $L_{2}$ be reduced by interposing the specimen $B$. Then $I_{2}$ will be less than $I_{1}$, and, with $U$ broken, the spot of light will be deflected. Two methods may be employed to make $I_{1}$ and $I_{2}$ again equal, a means of obtaining a measure of the transmission of $B$ being thus possible: ( $I$ ) The amount of radiant energy falling on $P_{1}$ may be decreased by narrowing $S_{1}$ until $I_{1}$ becomes equal to $I_{2}$, the ratio of the slit widths in the two cases being a measure of the transmission; or (2) the amount of radiant energy falling on $P_{2}$ may be increased by moving $L_{2}$ nearer the slit $S_{2}$ until $I_{2}$ again becomes equal to $I_{1}$, the inverse ratio of the squares of the distances in the two cases giving the transmission.

The value of the transmission obtained by the two methods will be the same only in case the dark currents are exactly equal and in case the two photoelectric cells obey exactly the same irradiation-current law. In case either of these conditions is not fulfilled, the value of the transmission obtained by the first method will be in error, but by the second method the accuracy of the value of the transmission will be unimpaired though either or both of these conditions are not fulfilled. Therefore, as is illustrated below, the transmission is measured by merely varying the distance of $L_{2}$ from the slit $S_{2}$, this distance being the only variable in the operation; for the width of the slit $S_{1}$, the amount and quality of the radiant energy falling on $P_{1}$ and $P_{2}$, the photo- 
electric currents $I_{1}$ and $I_{2}$, and the currents through $L_{1}$ and $L_{2}$ are unchanged, whether the specimen is in or out.

The following is the procedure used in measuring the transmission of the specimen $B$ : At the start the electrometer disk is charged by throwing the switch $K$, and the equality of the dark currents through $P_{1}$ and $P_{2}$ tested by breaking the ground connection of the electrometer quadrants at $U$. Then the lamps $L_{1}$ and $L_{2}$ are lighted and the currents adjusted to obtain the desired balance when the carrier is in the position C-"specimen out." This exact balance of photoelectric currents is obtained by bringing the light on the scale $D$ to rest, not necessarily at the zero position but in a position of no motion, which indicates that the electrometer quadrants are receiving no further charge; that is, $I_{1}=I_{2}$. Let this distance of $L_{2}$ from $S_{2}$ with the specimen out be $d_{\Lambda}$. Then the carrier is moved to position $C^{\prime}$, bringing the specimen into position in the path of the radiant energy from $L_{2}-$ "specimen in"-and, with $S_{1}$ and all other factors unchanged, $L_{2}$ is moved forward until a balance is again obtained, as indicated by the zero motion of the spot of light from the electrometer. Let this distance of $L_{2}$ from $S_{2}$ with the specimen in be $d_{\mathrm{B}}$. As a check, the specimen is again taken out (carrier back to $C$ ) and $L_{2}$ moved back till a balance is again obtained. This third reading $d_{\mathrm{A}}$ should check the first. It usually agrees to within a few millimeters, and the average is used. If, as is seldom the case, it differs by two centimeters or more, the readings are usually repeated. This depends on the accuracy desirable. Thus three readings are taken at each wave length, one with the specimen in and two with the specimen out, the transmission being equal to $\left(d_{\mathrm{B}} / d_{\mathrm{A}}\right)^{2}$. At low transmissions, since it is desirable to make sure that $d_{\mathrm{B}}$ can be obtained, the readings are usually taken in the order $d_{B}, d_{A}, d_{B}$, the rotating sector being started if it is impossible to obtain $d_{A}$ otherwise. For values of transmission of about 0.05 or less, the value of $d_{\mathrm{A}}$ is always obtained with the sector rotating, the ratio $\left(d_{\mathrm{B}} / d_{\mathrm{A}}\right)^{2}$ being multiplied by the proper factor to obtain the transmission. More readings can, of course, be taken if extreme accuracy is desired, but the above set of three, comprising a single determination, gives sufficient accuracy for nearly all kinds of work. The average time for a set of three readings is from 2 to 5 minutes, and the first set can usually be obtained within Io minutes after entering the room.

The operating current of $L_{2}$ is practically always within the range $17.0-19.5$ amperes, 19.5 being set as the upper limit 
in order to prolong the life of the lamp (rated at 20 amperes). The current $i_{2}$ (through $L_{2}$ ) might be kept at 19.5 amperes at all wave lengths and $i_{1}$ (the current through $L_{1}$ ) varied until the desired balance is obtained. However, this results in the blue in a sensitivity greater than is necessary. Therefore, it has been found practicable to make $i_{2}$ about 17.0 amperes at $460 \mathrm{~m} \mu$, making $i_{1}$ of such value as to bring about the desired balance. For wave lengths greater or less than $460 \mathrm{~m} \mu$, the value of $i_{1}$ is kept constant and $i_{2}$ increased until, at about 4 ro and $540 \mathrm{~m} \mu$, $i_{2}$ is made equal to 19.5 amperes. When measurements are made at wave lengths greater or less than these values, $i_{2}$ is kept at

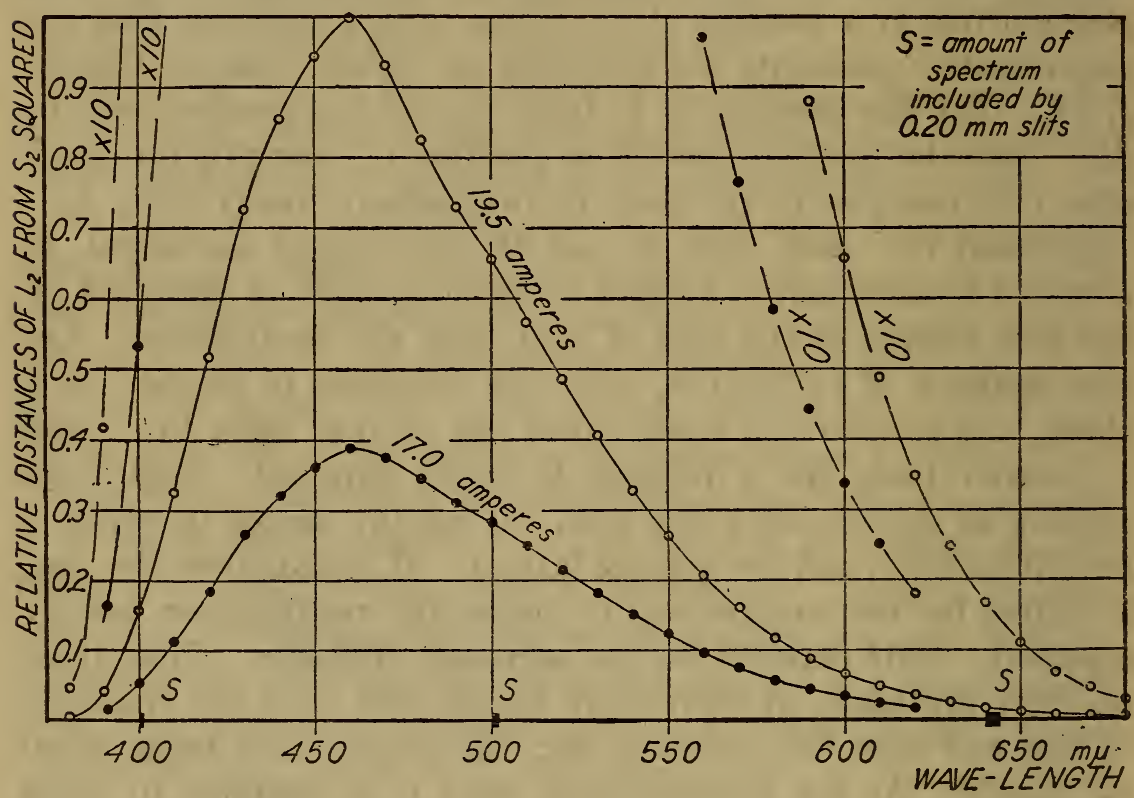

FIG. 2.-Curves showing spectral response of cell $P_{2}$, for currents of $I 9.5$ and $I 7.0$ amperes through $L_{2}$, under conditions of apparatus shown in Fig. $I$

19.5 amperes and $i_{1}$ lowered to get the desired balance. For low transmissions, higher values of the ratio $i_{2} / i_{1}$ become necessary. Thus a constant sensitivity is usually used for all wave lengths between 4 ro and $540 \mathrm{~m} \mu$, but at wave lengths greater or less than these limits a lowered sensitivity becomes necessary. However, lowered sensitivity means not entirely a decrease in the accuracy of the measurements, but, in part, merely an increase in the time required to obtain them.

In Fig. 2 are shown what might be called the spectral response curves of cell $P_{2}$, for currents of 19.5 and 17.0 amperes through $L_{2}$, under the particular concitions obtaining with this apparatus; 
that is, they are as actually obtained, uncorrected in any way, for the variable absorption or dispersion of the prism or the variable spectral distribution of radiant power from $L_{2}$. The ordinates are proportional to the squares of the distances of $L_{2}$ from $S_{2}$ necessary to obtain a balance, $i_{1}$ and $S_{1}$ being kept constant. The curves are doubtless a little high at the extremities, due to stray radiant energy from the blue or green regions. Stray ultra-violet radiant energy is effectually excluded by the absorption of the large glass prism, to which is also mainly due the sharp decrease in the response from 460 to $380 \mathrm{~m} \mu$.

\section{ERRORS AND ACCURACY}

As already stated, certain errors ordinarily met with when the photoelectric cell and electrometer are used to measure spectral transmission are eliminated by the null method herein described. These are:

I. Errors due to the photoelectric current not being strictly proportional to the radiant power incident on the cell. No calibrations of the cells are necessary, and it makes no difference what the relation is between radiant power and photoelectric current.

2. Errors due to what is ordinarily known as the dark current. It makes no difference whether or not it is eliminated. Nor do the dark currents through the two cells have to be the same, though approximately this condition is desirable for convenience' sake. It is necessary merely that conditions be steady over a period of from two to five minutes, the time usually required for a set of readings. Actually, conditions seem to be practically steady and unchanging for days, even weeks, at a time. In spite of this, any slight continuous change in conditions would tend to be nullified by the procedure of taking the first and third readings with the specimen removed and using the average.

3. Errors due to the use of the electrometer as a deflection instrument. Such errors may be those due to the deflections of the electrometer disk as given by the spot of light on the scale not being strictly proportional to the charge or potential acquired by the quadrants, those due to leakage of charges because of imperfect insulation or moist air, or those connected with the use of timing devices.

The errors which may be present can be put into two classes, those resulting from this method and apparatus and those to 
which all methods of spectrophotometry are liable. The errors resulting from this method and apparatus are of three kinds:

I. Those due to slight variations in the currents through the two lamps. In spite of the use of storage batteries, there are often small variations in the currents of one or both lamps, usually the large one, due probably to variations in the contact resistances by which the currents are regulated. If the first and third readings of a set disagree by more than $2 \mathrm{~cm}$, it is nearly always found to be due to one current or the other having varied and for the moment escaped observation. However, by means of the ammeters the currents are kept as nearly constant as it is possible to judge by the eye, and usually the third reading will check the first to I $\mathrm{cm}$ or better. And as already noted, the method of averaging the first and third readings tends to eliminate any errors due to gradually changing conditions.

2. Those due to the inverse-square law not being exactly obeyed by $L_{2}$. This would be expected to be very small because of the filament occupying an area of only $\mathrm{I} \mathrm{cm}^{2}$ and because it is not possible to move the filament closer to the slit than $46 \mathrm{~cm}$. It has been tested by means of the rotating sector. Other investigators ${ }^{8}$ have found that Talbot's law holds for the photoelectric cell; and a great number of observations at different times and at many wave lengths on the apparatus herein described prove that the inverse-square law is obeyed, assuming Talbot's law to hold, or that Talbot's law is obeyed, assuming the inverse-square law to hold. Actually, the error due to any failure of $L_{2}$ to obey the inverse-square law is too small to be detected over the range of distances used. It might be noted here that when the transmission of thick specimens is measured, correction is made to the value of $d_{\mathrm{B}}$ as read on the scale, this reading being larger than the true optical distance of $L_{2}$ from $S_{2}$.

3. Errors of observation, mainly those due to a natural tendency to hurry and not wait for the spot of light to come absolutely to rest. Two classes of work might be distinguished in this connection; first, measurements where only ordinary accuracy is desired, as in most tests and in other work where information as to the spectral transmission merely is desired; and secondly, measurements of a higher accuracy, where fine specimens are being standardized or where different methods are being com- 
pared by means of standard specimens or a rotating sector. The difference in the two cases is partly that of the time used in making the observation, higher accuracy being reached by waiting a longer time to see if the spot of light is truly at rest, and partly that of the number of readings in a set, more readings being taken for greater accuracy; e. g., the first, third, fifth, and seventh with the specimen out, the second, fourth, and sixth with it in, averages being used in computing the transmission.

The errors to which all methods of spectrophotometry are liable are those connected with the dispersion apparatus. These may be due to:

I. Stray radiant energy. As already mentioned, special diaphragms and, when needed, selective ray filters eliminate this error so far as can be detected.

2. Inaccurate wave-length calibration. The scale of the Hilger constant-deviation spectrometer is already calibrated in wave lengths with considerable accuracy. This calibration was very carefully checked visually from 390 to $600 \mathrm{~m} \mu$ by means of the Fraunhofer lines and a curve of corrections plotted, which is used in making wave-length settings. This correction does not amount to more than $0.7 \mathrm{~m} \mu$ over this range. It was rechecked photoelectrically by means of the mercury lines after the spectrometer was set in place. Errors in the wave-length settings are believed to be not greater than $0.2 \mathrm{~m} \mu$ in the blue and 0.5 $\mathrm{m} \mu$ in the red.

3. The use of finite slit widths in the spectrometer. Errors introduced for this reason are minimized by making the two slits $S_{2}$ and $S_{3}$ equal and constant for any set of readings. The amount of the spectrum included at different wave lengths by slit widths equal to $0.20 \mathrm{~mm}$ is indicated in Fig. 2. Higher accuracy might be reached by reducing the slit widths from 0.20 to $0.10 \mathrm{~mm}$, but with the present voltages on the cells this would, except in the blue, necessitate lowering the sensitivity, and would thus decrease the accuracy, as well as the range, at which it is possible to make measurements. Except for very narrow bands, this error due to finite slit widths is considered negligible for constant values of 0.20 $\mathrm{mm}$ or less. By raising the voltages on $P_{1}$ and $P_{2}$, keeping the proper value of the ratio $V_{2} / V_{1}$, the sensitivity would be increased, and it is probable that, with sufficiently higher voltages, the slits $S_{2}$ and $S_{3}$ could be reduced to o.1o $\mathrm{mm}$ with a resulting sensitivity as great as at present used. However, it is not desirable to get 
too near the critical voltage of the cells, and so far it has not seemed necessary to use voltages higher than those given. Further study of this question is planned.

The accuracy of the transmission measurements obtainable by this method and apparatus has been tested mainly in two ways:

I. By measuring the transmission of rotating sectors of known aperture.

2. By measuring the transmission of standard samples and other good specimens whose transmission has also been obtained by other methods, visual and photographic.

Further confidence in the method has been obtained as follows:

I. By measuring the transmission of two standard samples and using each of these in turn as a known comparison sample for obtaining the transmission of a third sample.

2. By measuring samples of different thicknesses and comparing observed with computed results, assuming Lambert's law.

3. By repeating measurements on the same specimen.

4. By the smoothness of transmission curves obtained.

So far as the errors resulting from this method are concernedviz, those due to possible variations in the currents through the lamps, those due to failure of $L_{2}$ to obey the inverse-square law, and those classed as errors of observation-their combined effect may be tested by means of measurements made on the rotating sectors. Tests have so far been made on sectors rated at 80,50 , and ro per cent transmission, the true transmissions having been determined by the photometric section of the Bureau as 0.800 , 0.500 , and 0.102 . Out of I2 measurements made on the 0.800 sector 8 were within 0.003 of this value, the greatest deviation being 0.007 . Out of 2 I measurements made on the 0.500 sector 16 were within 0.002 of this value, the greatest deviation being 0.008 . Out of 10 measurements made on the 0.102 sector 6 were within 0.0015 of this value, the greatest deviation being 0.003 . These measurements comprise all that have been made since the sectors were received, with the exception of those made on one lamp which did not stand vertically in the socket and which gave values from 0.005 to 0.015 too low. They have been obtained at a number of different times, on different lamps, by different observers, at wave lengths from 400 to $600 \mathrm{~m} \mu$, and with $L_{2}$ at distances from $S_{2}$ varying from $250 \mathrm{~cm}$ to $50 \mathrm{~cm}$. Not many of them are precision measurements, the majority being taken with the same ordinary care used in most transmission measurements. 
There is no consistent deviation from the inverse-square law when $L_{2}$ is anywhere from 50 to $250 \mathrm{~cm}$ from $S_{2}$. Conversely, as already noted, the above measurements add considerable proof that Talbot's law of the rotating sector is valid when applied to the photoelectric cell. The speed of rotation seems to make no difference.

However, the tests by means of the rotating sectors give no clue to the uncertainties or errors which are liable to be present in all methods of spectrophotometry; viz, those due to stray light, finite slit widths, etc. In fact, the only final test for any method is its agreement with other methods, due regard being paid to all the known factors of accuracy or error. Inasmuch as an investigation now being made into the agreements between the different methods at present used in the color-standardization work of the Bureau has not yet been completed, comparison is made in this paper only with results obtained visually on the Martens polarization photometer ${ }^{9}$ at those wave lengths where monochromatic light can be obtained. The mercury arc has been used as a source, the violet, blue, green, and yellow lines being isolated by suitable color screens, giving practically monochromatic light at $406,436,546$, and $578 \mathrm{~m} \mu$. Inasmuch as all questions of stray light and slit widths are avoided in this instrument, it is a valuable means of comparison. However, if steep transmission curves happen to be encountered at 406 or $578 \mathrm{~m} \mu$, then, of course, uncertainty enters, since there are in reality two lines close together at approximately these wave lengths. It should be noted also that, since the transmission is equal to $\tan \theta_{1} \cot \theta_{2}$, there results at low transmissions much greater accuracy in terms of the transmission unit than at high transmissions. For example, the range of angle giving transmissions from 0.50 to $r .00$ is about $35^{\circ}-45^{\circ}$ for $\theta_{1}$ and $45^{\circ}-55^{\circ}$ for $\theta_{2}$, but for transmissions from 0.00 to $0.5^{\circ}$ the range is $0^{\circ}-35^{\circ}$ and $55^{\circ}-90^{\circ}$, respectively. This makes little difference for green and yellow light, to which the eye is very sensitive; but for the blue and especially the violet mercury lines the uncertainty of measurement for high transmissions is considerable. It is thought, therefore, that discrepancies occurring at 436 and $406 \mathrm{~m} \mu$ are mainly due to errors on the Martens

\footnotetext{
- Phys. Zeit. I, p. 299; 1900 . In this instrument two beams of light from the same source are polarized mutually perpendicular by means of a Wollaston prism and brought together to form the two halves of a photometric field. This field is viewed through a nicol prism, and the adjustment is such that the two halves of the field are brought to equality by the analyzing nicol at about $45^{\circ}$ on the scale. The specimen was placed first in one beam and then in the other and the analyzing nicol rotated in each case until a photometric match was obtained. The tangent of the angle in one case multiplied by the cotangent in the other gives the transmission of the specimen; that is, $T=\tan \theta_{1} \cot \theta_{2}$.
} 
photometer. It is at 546 and $578 \mathrm{~m} \mu$, if the curves are not too steep at this latter wave length, that the most valuable checking can be done, for here the visual method is of great precision, while the photoelectric method is not at its best from the standpoint of either sensitivity or dispersion. Whatever accuracy and reliability, then, is found for the photoelectric method at these wave lengths should be equaled or surpassed throughout the whole range from these wave lengths to and including 4 Iо $\mathrm{m} \mu$.

A number of transmission ${ }^{10}$ curves are shown in Figs. 3 to 9. The Jena specimens whose curves are shown in Figs. 3 to 7 , with some

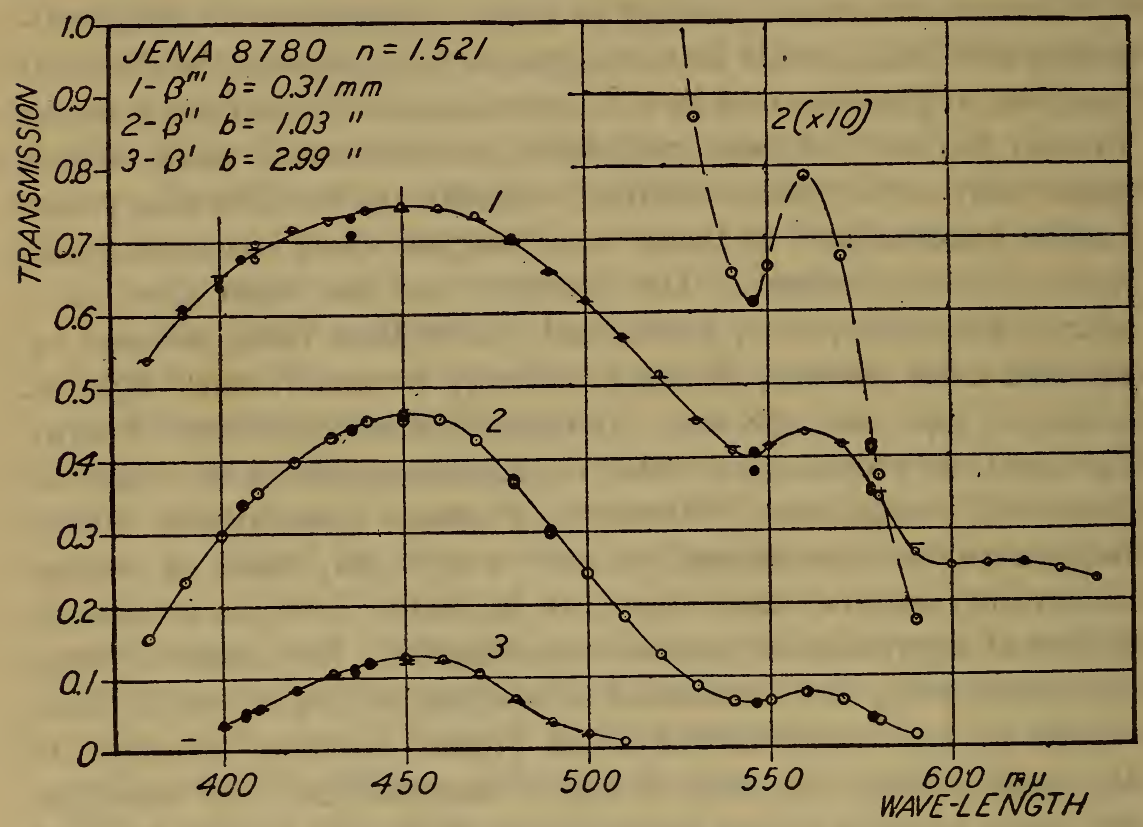

FIG. 3.-Transmission curves illustrating agreements between photoelectric and visual methods. For explanation see p. 24,346

red samples whose curves are not shown, comprise a set of standard precision glasses kept on file at the Bureau. These glasses, so far as can be detected, are free from all defects such as striæ, bubbles, etc., and have been finished with great care, the surfaces being highly polished and made parallel to within o.or $\mathrm{mm}$. They were prepared and are used mainly for a comparison of different methods in order that any discrepancies found may be laid entirely to the methods and not to the specimens. The other samples, Figs. 8 and 9, are considered as fairly good specimens, but were not

10 The transmission of a specimen (distance between surfaces $=b$ ) is defined as that fraction of radiant power incident on the first surface which gets through the second surface. 


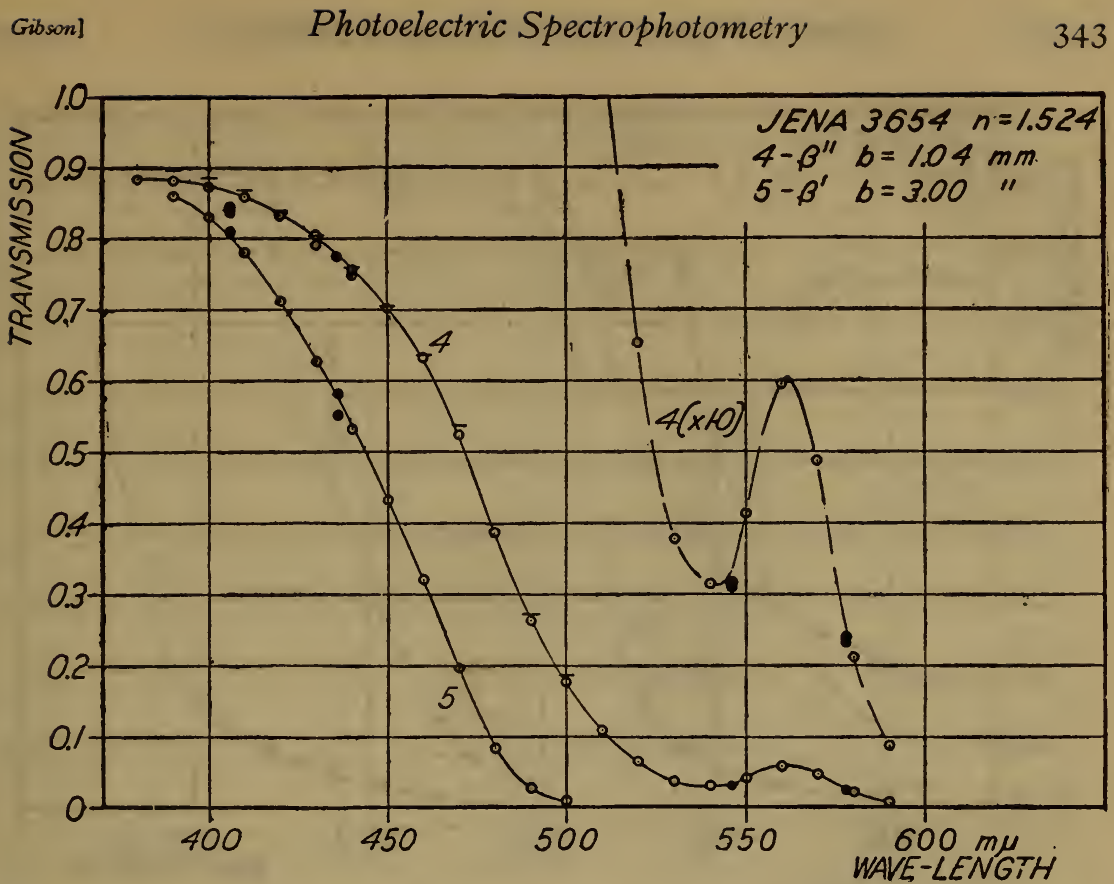

FIG. 4.-Transmission curves illustrating agreements between photoelectric and visual methods. For explanation see p. $24 \pi 34 \mathbb{F}^{*}$

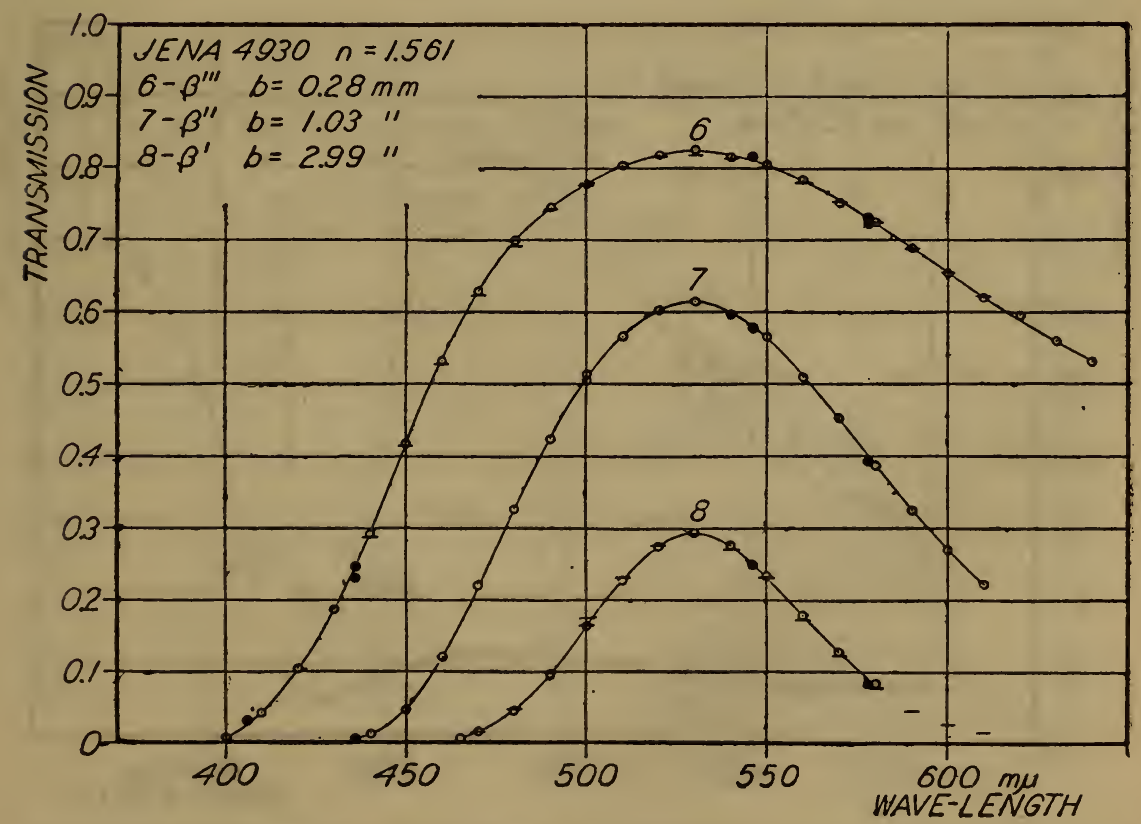

FIG. 5.-Transmission curves illustrating agreements between photoelectric and visual methods. For explanation see p. 2434 


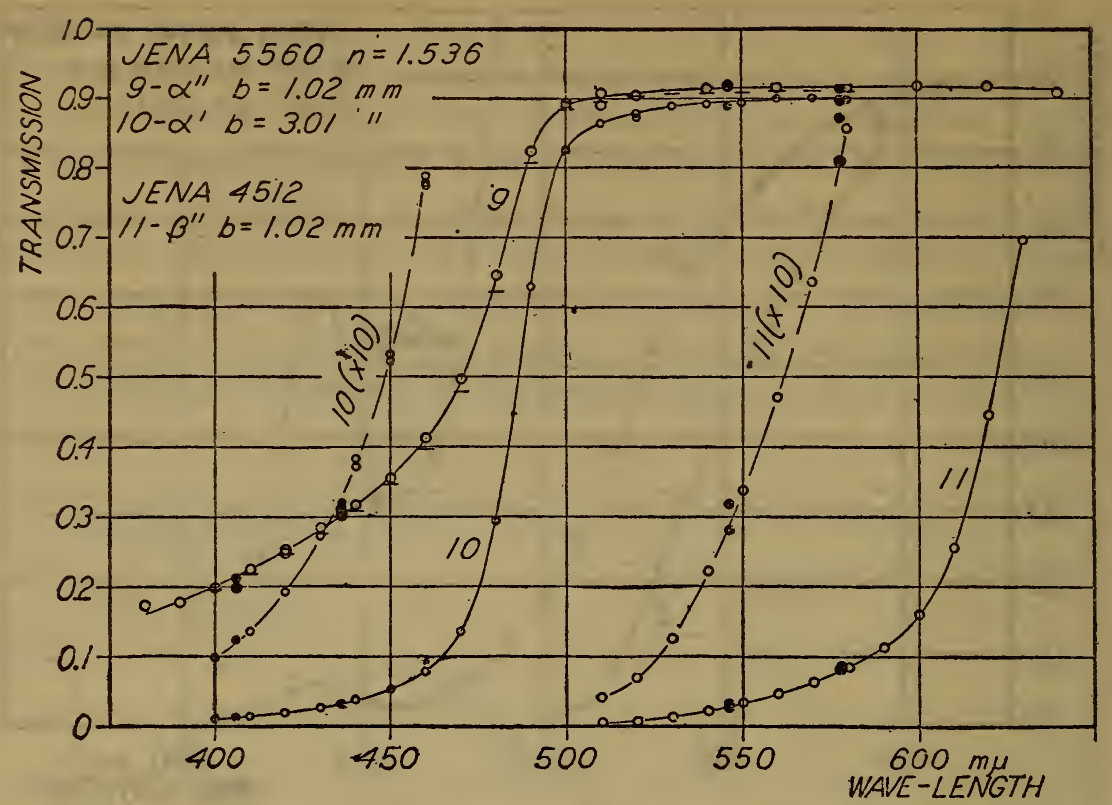

FIG. 6.-Transmission curves illustrating agreements between photoelectric and visual methods. For explanation see p. 24 aij 346

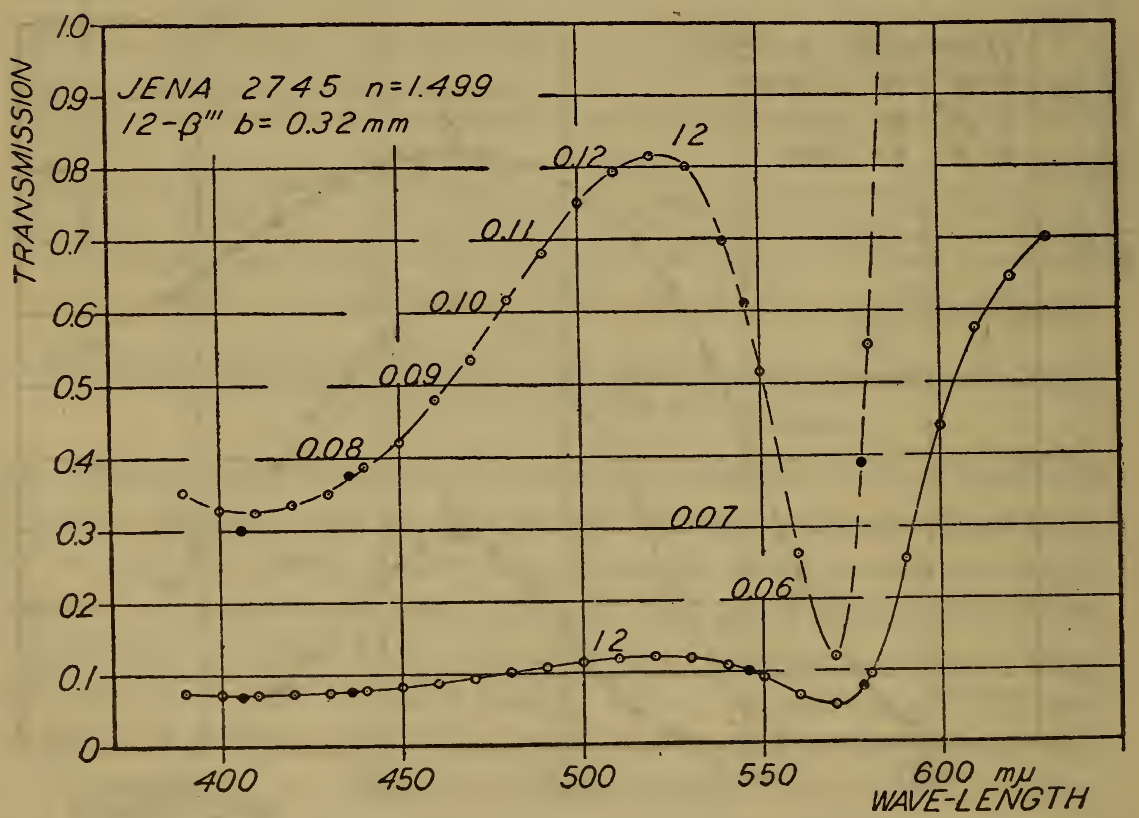

FIG. 7.-Transmission curves illustrating agreements between photoelectric and visual methods. For explanation see p. 24,3 섬 


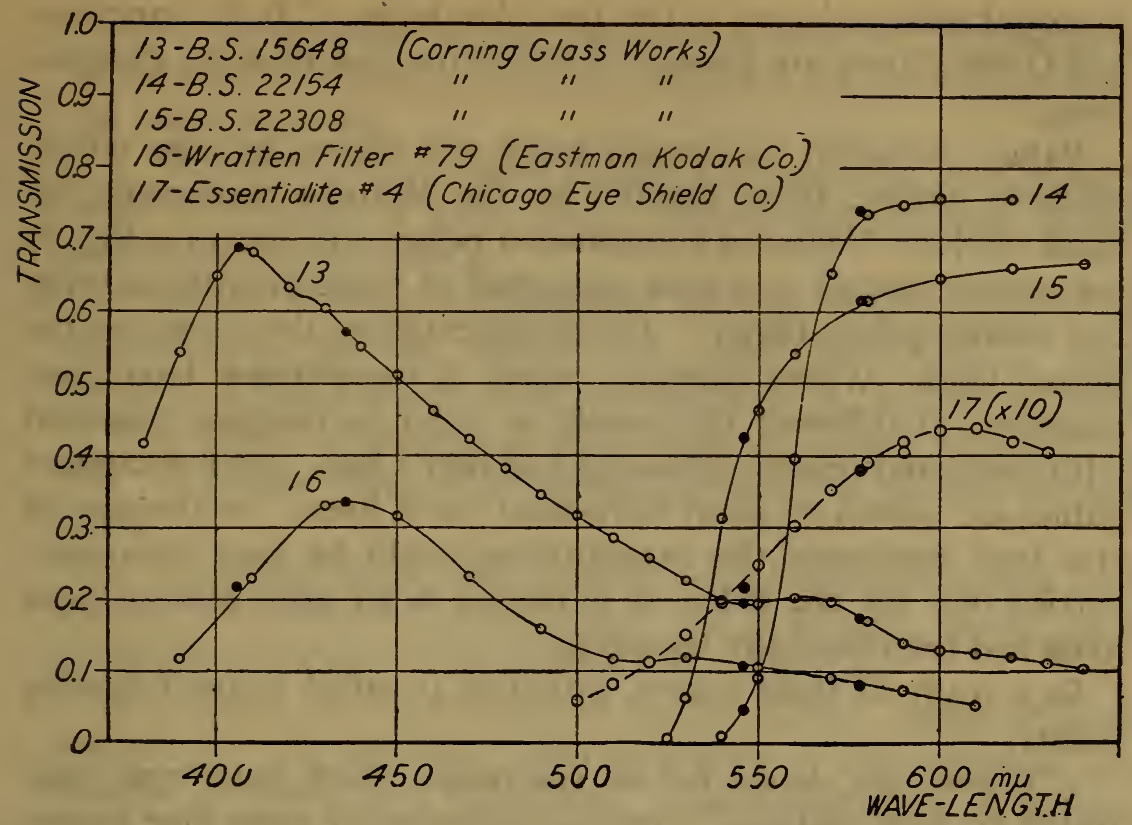

FrG. 8.-Transmission curves illustrating agreements between photoelectric and visual methods. For explanation see $p, 2846$

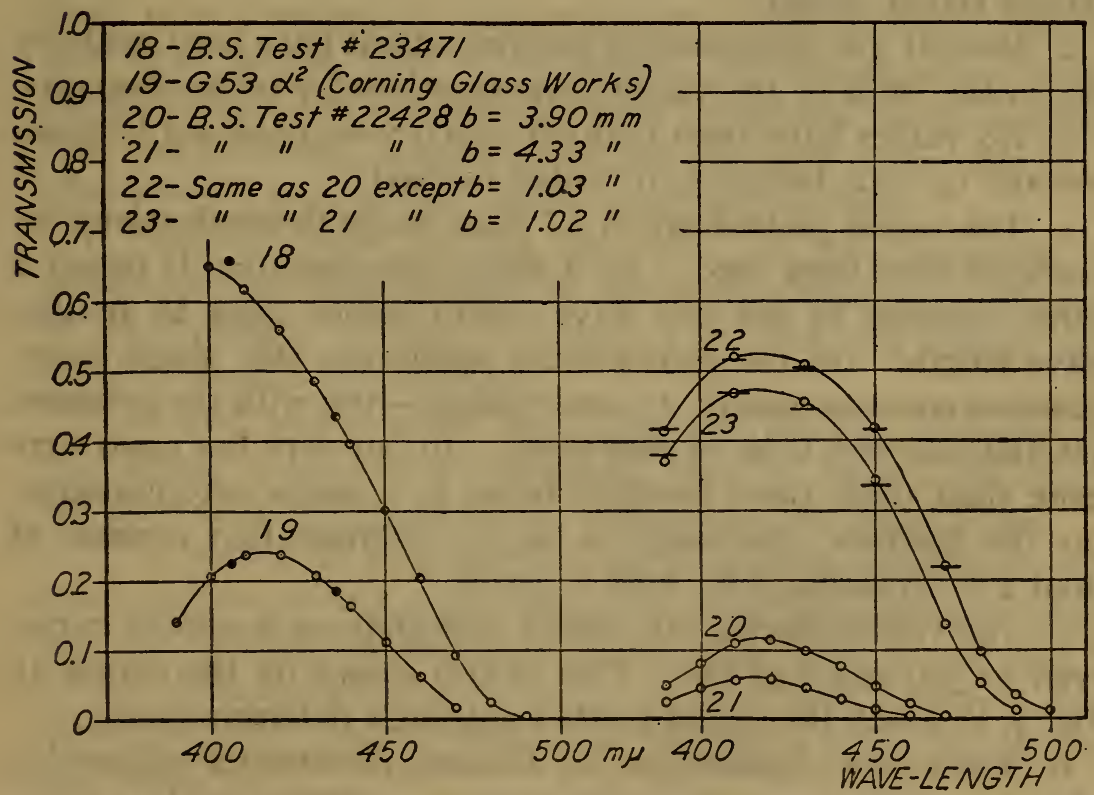

FIG. 9.-Transmission curves illustrating agreements between photoelectric and visual methods. For explanation see p. 24,346 
prepared with such care as the Jena standards. "B. S." numbers and Greek letters are Bureau of Standards, not makers', designations.

Values obtained photoelectrically are shown by the whitecentered circles; those visually on the Martens photometer by black circles. Values of transmission below 0.10 (or 0.12, Fig. 7) are usually plotted to a scale magnified ro times in order to bring out various points better. This is indicated on the curves by the dotted lines. Where possible, values of transmission have been measured at different thicknesses in order to compare observed with computed results, assuming Lambert's law. Such computed values are shown by short horizontal black lines. In the case of the Jena specimens this computation could be very accurately carried out, for the index of refraction $n$ for each kind of Jena glass had been carefully measured.

In a study of these curves, attention is called to the following points:

I. Curves are shown for all specimens which have ever been tested by both methods, with the exception of some blue glasses which have the same types of curves and which show the same sort of agreements or disagreements at 436 and $406 \mathrm{~m} \mu$ as the blue glasses herein shown.

2. Most of the photoelectric determinations have been made by the writer, most of the visual determinations by other observers.

3. No values have been omitted which were obtained between 380 and $640 \mathrm{~m} \mu$, inclusive, by either method.

4. The values plotted are in all cases single determinations; no averages have been used. By a single determination is meant a value obtained at any one wave length before going to another wave length. On the photoelectric apparatus this single determination consists usually of three readings-two with the specimen out and one with it in, or vice versa. In but very few cases were more than these three readings taken in a single determination. On the Martens photometer a single determination consists of from 2 to Io readings for both $\theta_{1}$ and $\theta_{2}$.

5. The values plotted fall almost uniformly on a smooth curve, even to 380 and $640 \mathrm{~m} \mu$. This is also shown by the curves in Fig. 2 , in which the values plotted are single determinations.

6. The values obtained on the Martens photometer seldom fall off this smooth curve by as much as o.or. Different determinations of the transmission obtained on this instrument show that even at 546 and $578 \mathrm{~m} \mu$ a discrepancy between methods is not 
entirely due to error in the photoelectric method. Discrepancies occurring at 406 and $436 \mathrm{~m} \mu$ are considered mainly due to error in the visual method.

7. Values below 0.15, when plotted to a magnified scale, show the same smoothness of curve and a discrepancy between methods seldom more than 0.0015 .

8. Beyond $600 \mathrm{~m} \mu$ an increasing error is often noticed in the photoelectric method (shown by comparison with a visual method, König-Martens spectrophotometer, of known reliability in this region) amounting in some cases to as much as 0.05 at $65^{\circ}$ $\mathrm{m} \mu$. A careful study of conditions beyond $600 \mathrm{~m} \mu$ has not been made.

9. Discrepancies between observed and computed results are of interest as a test of Lambert's law, but, in general, should not be considered as a test of the accuracy of a method.

In addition to the data given in the figures, values of transmission at $546 \mathrm{~m} \mu$ have been measured by each method on a number of fairly good specimens. The results were no different from those illustrated by the curves.

Besides the data herein given showing the kind of agreements obtained by these two methods, many other data are on file which have been obtained by other methods, visual and photographic, and which will in time be published. By a comparison with all these methods and after nearly a year's continual use, it is believed that the following statements of the accuracy obtainable by the photoelectric null method herein described are true:

I. Between wave lengths $4 \mathrm{IO}$ and $550 \mathrm{~m} \mu$, inclusive, the uncertainty of measurements is not greater than o.or for values of transmission between 0.00 and 1.00 , and not greater than 0.003 between 0.00 and $0.10 .^{11}$

2. Beyond this range as far as 390 and $600 \mathrm{~m} \mu$, inclusive, the uncertainty is not greatly increased, being at these wave lengths not more than twice as great as throughout the better range.

3. To state it in another way, the percentage uncertainty from wave lengths 4 Io to $550 \mathrm{~m} \mu$, inclusive, varies from not more than I per cent at high transmissions to not more than ro per cent for values of transmission equal to o.or or less, with slightly increasing uncertainty beyond these wave lengths as far as 390 and 600 $\mathrm{m} \mu$, inclusive.

11 To illustrate: If transmission values equal to 0.793 or 0.0396 be obtained, it is believed that the true values can be guaranteed to be within the values 0.783 to 0.803 or 0.0366 to 0.0426 , respectively. This method of stating the uncertainty is followed throughout the paper, except in paragraph 3 on this page. 


\section{DIFFUSE SPECTRAL REFLECTION}

The diffuse spectral reflection of substances is measured relative to that of some "white" substance, such as magnesium carbonate, taken as a standard and given the value of $\mathrm{I} .00$ for the reflection at all wave lengths. The changes necessary in transforming the apparatus to the measurement of reflection instead of transmission may be explained by means of Fig. Io. The lamp $L_{2}$ (Fig. I), which for its minimum distance from the slit $S_{2}$ is in the position of $L_{2}$ (Fig. Io) for transmission measure-

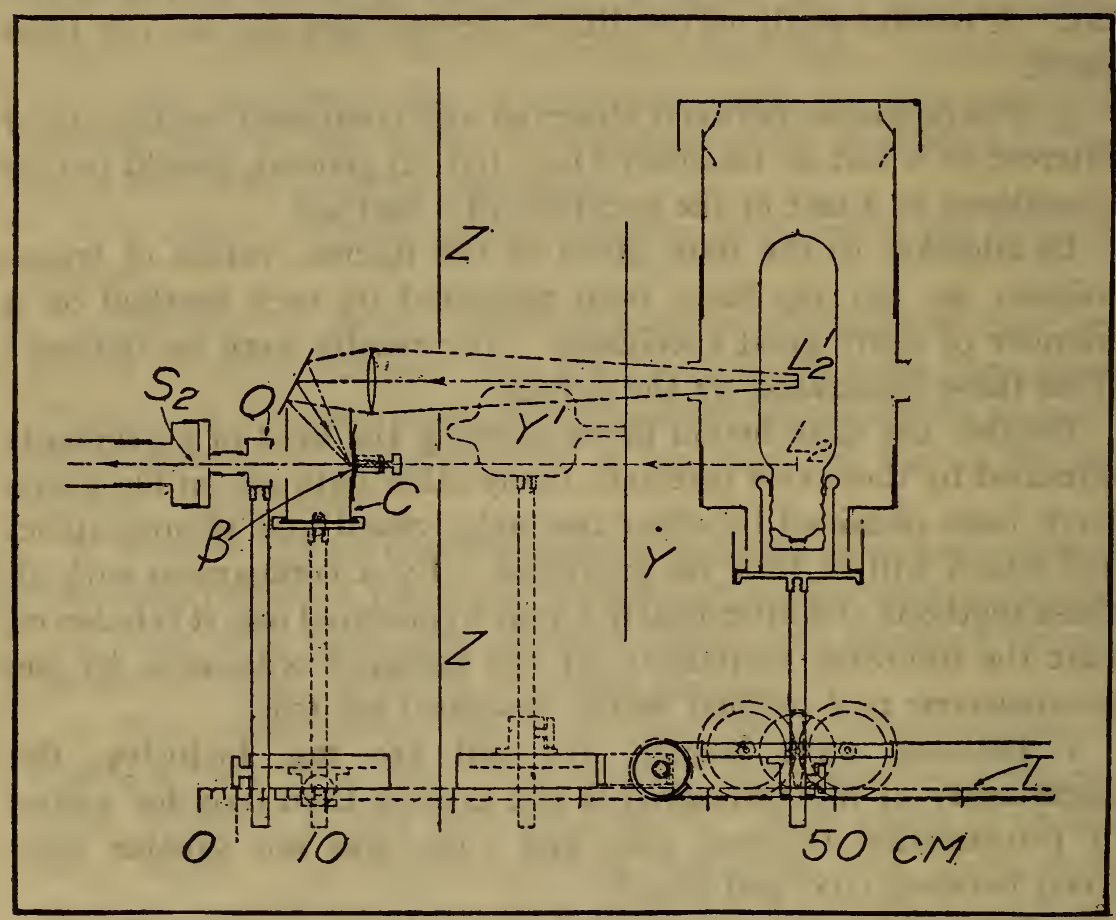

FIG. Io.-Illustrating changes in apparatus necessary for measuring diffuse spectral reflection

ments, is raised to the position of $L_{2}^{\prime}$ and kept in that position without further change. The hard-rubber holders which keep the specimen $B$ (Fig. I) in position are removed from the carrier $C$. The material whose reflection is to be studied is held firmly in position over one of the openings in $C$ as indicated at $\beta$ (Fig. 10), being pressed firmly and uniformly against the outside of $C$ by means of a suitable clamp fastened to $C$. The comparison substance is clamped in position over the outside of the other opening in $C$ (not shown in Fig. ro). By means of a 
lens and mirror, as shown, the radiant energy from $L_{2}{ }^{\prime}$ is brought to a focus on the surface of the reflecting substance at $\beta$, in front of $S_{2}$. In short, the arrangement is such that either the standard substance or that whose reflection is to be measured may be brought, by moving the carrier $C$ back and forth, into position in exactly the same place in the focus of the radiant energy from $L_{2}^{\prime}$ and directly in front of the slit $S_{2}$. The diffuse reflection in the direction of $S_{2}$ enters the spectrometer and reaches the cell $P_{2}$ as before, the photoelectric current thus excited being balanced by the current from $P_{1}$ due to $L_{1}$. The observer takes the position $X^{\prime \prime}$ (Fig. I), where he can vary the width of $S_{1}$ while watching the spot of light, the mirror and scale being at $M^{\prime \prime}$ and $D^{\prime \prime}$. As before, an assistant is at $X^{\prime}$, where he can watch the currents, manipulate the carrier $C$, and adjust the wavelength scale of $H$.

To obtain the value of the diffuse reflection, the following readings are taken: First, the standard magnesium-carbonate surface is brought into position before $S_{2}$ in the focus of the radiant energy from $L_{2}{ }^{\prime}$, and $S_{1}$ varied to obtain a balance. Then the sample whose reflection is to be measured is brought into position by moving the carrier $C$, and $S_{1}$ varied to get a new balance. Finally, a check value of $S_{1}$ is taken with the standard back in position, and the ratio of the value of $S_{1}$ with the sample in position to the average of the values of $S_{1}$ when the standard is in position is taken as the reflection of the sample relative to that of the standard.

As so far used, the slits $S_{2}$ and $S_{3}$ have usually been made 0.25 or $0.30 \mathrm{~mm}$ wide, although for values of reflection below 0.05 , as well as at $390 \mathrm{~m} \mu$, they are usually made 0.40 or 0.50 $\mathrm{mm}$; and at $460 \mathrm{~m} \mu$, when the magnesium-carbonate surface is before $S_{2}$, the value of $S_{1}$ necessary for a balance is from $\mathrm{r} .50$ to $2.00 \mathrm{~mm}$, the currents through the lamps being the same as before. Under these conditions the sensitivity is thus approximately the same as that used in measuring transmission. There is this difference, however. In the method used for transmission measurements a constant sensitivity is used throughout a single determination-that is, whether the specimen is in or out-but in the method just described for reflection the sensitivity depends on the reflection, and thus varies in a single determination. This is unimportant except at very low reflections.

This is still a null method and avoids all errors connected with electrometer deflection methods. But; as already mentioned, 
errors will be introduced in this use of the null method if the two photoelectric cells do not obey the same current-irradiation law and if the two dark currents through $P_{1}$ and $P_{2}$ are not exactly balanced. As previously explained, the dark currents may be balanced by adjusting the ground connection $G^{\prime}$ (Fig. I) until the ratio $V_{2} / V_{1}$ is of the proper value. That the two photoelectric cells obey practically the same current-irradiation law has been proved at different times, with the apparatus as in Fig. I, by testing the relation between values of the squares of the distances of $L_{2}$ from $S_{2}$ and the corresponding values of the width of $S_{1}$ necessary to obtain a balance as shown by the electrometer. The results show that for a balanced condition the width of the slit $S_{1}$ is directly proportional to the squares of the distances of $L_{2}$ from $S_{2}$; that is, there is a straight-line relation. When plotted, the values of $S_{1}$ show no consistent deviation from this straight line, being never more than $0.01 \mathrm{~mm}$ from it for values between 0.30 and $2.00 \mathrm{~mm}$. At $0.20 \mathrm{~mm}$ and below, this deviation is occasionally, though not always, as much as $0.02 \mathrm{~mm}$. Above $2.00 \mathrm{~mm}$, it has not been tried.

The method has not been as thoroughly tested as that for transmission measurements. A few tests have been made, however, on various substances, and a comparison with other methods, visual and photographic, has been thus possible. It is believed that the following statements are true in regard to the reflection of completely diffusing substances relative to that of some second completely diffusing material like magnesium carbonate:

I. Between 410 and $550 \mathrm{~m} \mu$, inclusive, the uncertainty of values obtained is not greater than 0.02 for values of relative reflection between 0.00 and 1.00 , and not greater than 0.01 between 0.00 and 0.20 .

2. This uncertainty increases somewhat as wave lengths 390 and $600 \mathrm{~m} \mu$ are reached, being perhaps twice as great as throughout the better range.

\section{OTHER APPLICATIONS}

The apparatus as herein described is well adapted for the comparison of the spectral distribution of radiant power of two sources over the same range of wave lengths as used in transmission and reflection measurements. If the two sources are such that they obey the inverse-square law, the method of varying their distances from $S_{2}$ could be used, $S_{1}$ being kept constant: If the inverse-square law were not obeyed by either source, the other 
method could be used, their distances from $S_{2}$ being kept constant and $S_{1}$ varied to obtain a balance. The accuracy would be the same, respectively, as that already stated for the measurement of transmission or reflection. For greatest accuracy a device should be made by which first one and then the other source could be moved into position, check readings being taken on one source before and after the reading on the other. For lack of such a device, curves similar to those of Fig. 2 for each source could be obtained by varying the distance of the source from $S_{2}$ or by varying the width of $S_{1}$, and the ratio of the ordinates of the two curves, wave length by wave length, would give the relative spectral distribution of radiant power. If one of the two lamps is a standard whose radiant power curve is known, then, of course, the actual distribution curve for the other lamp could be computed.

Another use to which this apparatus could be put would be the measurement of fluorescence. If a curve similar to that of Fig. 2 were obtained for a source whose spectral distribution of radiant power is known, this curve could be reduced to that for a source emitting equal amounts of energy at all wave lengths. With the apparatus arranged as in Fig. Io, the fluorescent material could be held in the position of the reflecting surface $\beta$, and the exciting radiant energy, which, of course, must be of constant intensity and of wave length different from that of the fluorescence, focused upon it. The values of $S_{1}$ necessary for a balance could then be obtained at each wave length; and if these values were divided by the values for the equal-energy source, the result would be the amount of energy emitted by the fluorescent substance at each wave length.

As already mentioned, the sudden decrease of the sensitivity as wave lengths shorter than $460 \mathrm{~m} \mu$ are encountered is largely due to the absorption of the large glass prism in the Hilger constant-deviation spectrometer, the potassium-hydride surface being very sensitive to the ultra-violet. There is, of course, nothing about the method which would prevent its extension into the ultra-violet, if a spectrometer with quartz prism and lenses were used in place of the present instrument. The windows $W_{1}$ and $W_{2}$ (Fig. I) are made of quartz, and photoelectric cells can be obtained made entirely of quartz or with quartz windows. For wave lengths shorter than about $300 \mathrm{~m} \mu$, a different source would have to be used because of the absorption in the thin glass bulb. 


\section{SUMMARY}

Reliable determinations of spectral transmission throughout the green, blue, and violet have been made by means of the photoelectric null method as herein described.

This apparatus has been in continual use since April, 1918, and has proved very satisfactory as regards speed of operation, ease of keeping in proper working condition, and accuracy of measurement.

All errors have been eliminated, as well as the necessity of any tests, calibrations, or corrections, in connection with the currentirradiation law or the dark currents of the photoelectric cells, or with electrometer deflection methods.

The only variable in the process of making a determination of the transmission at any wave length is the accurately measurable distance of a small light source from the slit of the spectrometer.

Because of the higher sensitivity of the electrometer, it has been possible to make measurements over a greater range and, at least for low values of transmission, with greater accuracy than could be made if a galvanometer were used.

The accuracy has been tested by measuring the transmission of rotating sectors of known aperture and the transmission of standard glasses which have been measured by other methods, visual and photographic. It is believed the following statements are true:

I. Between wave lengths 4 ro and $55^{\circ} \mathrm{m} \mu$, inclusive, the uncertainty of measurements is not greater than o.or for values of transmission between 0.00 and $\mathrm{r} .00$, and not greater than 0.003 for values between 0.00 and 0.10 .

2. Beyond this range as far as 390 and $600 \mathrm{~m} \mu$ the uncertainty is slightly greater, being at these wave lengths perhaps twice as great as throughout the better range.

Measurements of diffuse spectral reflection relative to that of magnesium carbonate have been made, comparison with other methods indicating an uncertainty, between wave lengths 4 ro and $55^{\circ} \mathrm{m} \mu$, inclusive, not greater than 0.02 for values of relative reflection between 0.00 and 1.00 , and not greater than 0.01 for values between 0.00 and 0.20 , this uncertainty becoming perhaps twice as great as wave lengths 390 and $600 \mathrm{~m} \mu$ are reached.

The method is also applicable to the measurement of the relative radiant power of two sources and to the measurement of fluorescence, and could be extended into the ultra-violet if quartz parts were used instead of glass.

WASHINGTON, February I 2, I919. 